UCRL-JC-126730

PREPRINT

\title{
Treatment of Patient-Dependent Beam Modifiers in Photon Treatments by the Monte Carlo Dose Calculation Code PEREGRINE
}

A. E. Schach von Wittenau, L. J. Cox, P. M. Bergstrom Jr., S. M. Hornstein, R. Mohan, B. Libby, Q. Wu, D. M. J. Lovelock

This paper was prepared for submittal to the

12th International Conference on the Use of Computers in Radiation Therapy

Salt Lake City, UT

May 27-30, 1997

March 4, 1997

This is a preprint of a paper intended for publication in a journal or proceedings. Since changes may be made before publication, this preprint is made available with the understanding that it will not be cited or reproduced without the permission of the author. 


\section{DISCLAIMER}

This document was prepared as an account of work sponsored by an agency of the United States Government. Neither the United States Government nor the University of California nor any of their employees, makes any warranty, express or implied, or assumes any legal liability or responsibility for the accuracy, completeness, or usefulness of any information, apparatus, product, or process disclosed, or represents that its use would not infringe privately owned rights. Reference herein to any specific commercial product, process, or service by trade name, trademark, manufacturer, or otherwise, does not necessarily constitute or imply its endorsement, recommendation, or favoring by the United States Government or the University of California. The views and opinions of authors expressed herein do not necessarily state or reflect those of the United States Government or the University of California, and shall not be used for advertising or product endorsement purposes. 


\title{
Treatment of Patient-Dependent Beam Modifiers in Photon Treatments by the Monte Carlo Dose Calculation Code PEREGRINE
}

\author{
A. E. Schach von Wittenau ${ }^{1}$, L. J. Cox ${ }^{1}$, P. M. Bergstrom Jr. ${ }^{1}$, S.M. Hornstein ${ }^{1}$, \\ R. Mohan ${ }^{2}$, B. Libby ${ }^{2}$, Q. Wu ${ }^{2}$, D. M. J. Lovelock ${ }^{3}$, \\ ${ }^{1}$ Lawrence Livermore National Laboratory, Livermore, CA; \\ ${ }^{2}$ Medical College of Virginia, Richmond, VA; \\ ${ }^{3}$ Memorial Sloan Kettering Cancer Center, NY, NY
}

The goal of the PEREGRINE Monte Carlo Dose Calculation Project [1] is to deliver a Monte Carlo package that is both accurate and sufficiently fast for routine clinical use. One of the operational requirement for photon-treatment plans is a fast, accurate method of describing the photon phase-space distribution at the surface of the patient.

The open-field case is computationally the most tractable; we know, a priori, for a given machine and energy, the locations and compositions of the relevant accelerator components (i.e., target, primary collimator, flattening filter, and monitor chamber). Therefore, we can precalculate [2] and store [3] the expected photon distributions. For any open-field treatment plan, we then evaluate these existing photon phase-space distributions at the patient surface, and pass the obtained photons to the dose calculation routines within PEREGRINE. We neglect any effect of the intervening air column, including attenuation of the photons and production of contaminant electrons.

In principle, for treatment plans requiring jaws, blocks, and wedges, we could precalculate and store photon phase-space distributions for various combinations of field sizes and wedges. This has the disadvantage that we would have to anticipate those combinations and that subsequently PEREGRINE would not be able to treat other plans. Therefore, PEREGRINE tracks photons through the patient-dependent beam modifiers. The geometric and physics methods used to do this are described here.

\section{Geometric Methods}

As currently implemented in PEREGRINE, wedges, blocks, and jaws occupy separate vertical positions in the space between the bottom of the flattening filter and the uppermost surface of the patient. Thus, a photon's $z$ position determines whether it is in free space or inside a beam modifier. If the photon is in the free space between modifiers, it is simply translated to the top of the next modifier or to the patient surface. If it is at the top of a modifier, a path length step is generated at random, based on the material composition of the modifier. The photon is moved to this new location. If this new position is below the bottom surface of the modifier, the photon is moved to the next modifier or to the patient. On the other hand, if the photon is still within the modifier, then scattering interactions are sampled and secondary particles are banked, as appropriate, for later transport.

\section{Wedges}

Figure 1 shows a schematic wedge. A wedge is described by the vertices formed by the intersections of the planes that define the wedge

This work was performed under the auspices of the U.S. Department of Energy by the Lawrence Livermore National Laboratory under contract number W-7405-ENG-48. surface. A photon is translated, via the method described above, from the top of the wedge to a point $(x, z)$, which may or may not be within the wedge itself. If $z$ is below $z_{\text {bottom }}$, then the photon has transited the wedge. If $z$ is above $z_{\text {bottom }}$, then the photon is either in the wedge or in the air gap below the wedge but above $z_{\text {bottom. }}$ At this point PEREGRINE loops, over the $\left(\boldsymbol{x}_{\boldsymbol{i}}, z_{\boldsymbol{i}}\right)$ pairs defining the vertices. It determines which abscissae $\left(\boldsymbol{x}_{\boldsymbol{i}+1}, \boldsymbol{x}_{\boldsymbol{i}+1}\right)$ bracket the photon's current $\boldsymbol{x}$ position, and then determines if the current $(x, z)$ position is above or below the plane defined by $\left(x_{i}, z_{i}\right)$ and $\left(x_{i+1}, z_{i+1}\right)$. If the photon is determined to be above the plane (i.e., within the wedge), then scattering interactions are sampled. If the photon is determined to be below the plane (i.e., beyond the wedge), it is translated to the next modifier or to the patient surface.

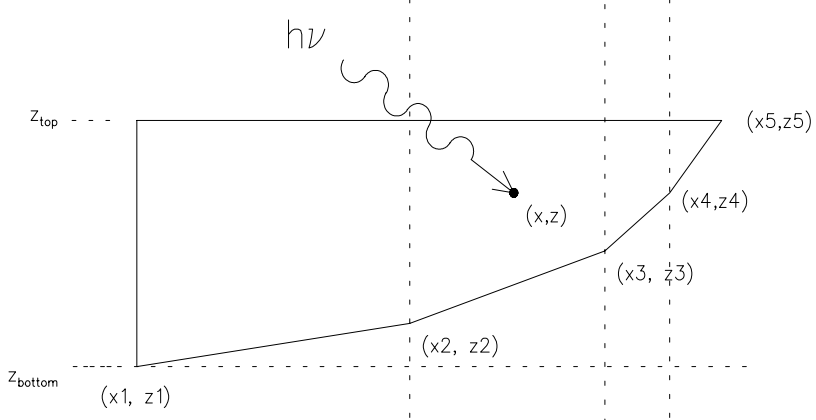

Figure 1: Schematic drawing of PEREGRINE's definition of a wedge. A wedge is defined by a top surface, a vertical extent, and a series of vertices describing the wedge surface planes. A photon is translated into the wedge. At this point, PEREGRINE determines which vertices bracket the photon's $\boldsymbol{x}$ position, and then determines if the photon is above the plane defined by these vertices (i.e., still within the wedge material) or below this plane (i.e., outside the wedge).

\section{Blocks}

PEREGRINE defines a block as a $500 \times 500$ mask array of square pixels corresponding to physical dimensions of $25 \times 25 \mathrm{~cm}^{2}$ at the patient surface. A pixel is defined to be either open or blocked. This is shown schematically in Figure 2. As with wedges, a photon is transported to the top of the block, at which point a material-dependent step is taken. If the photon has stepped to below the bottom surface of the block, it has cleared the block and is translated to the next modifier or to the patient surface. If the photon has not cleared the block in this fashion, then it is either within the block material or within a hole within the block. PEREGRINE decides which is the case by translating (and scaling) the pixel mask to the photon's $z$ 
position (Figure 3). If the photon's $(\boldsymbol{x}, \boldsymbol{y})$ coordinates lie within a blocked pixel, the photon is taken to be within the block material and scattering interactions are sampled. If the pixel is open, the photon is taken to be in an unblocked region. No interaction occurs, and another random step is taken.

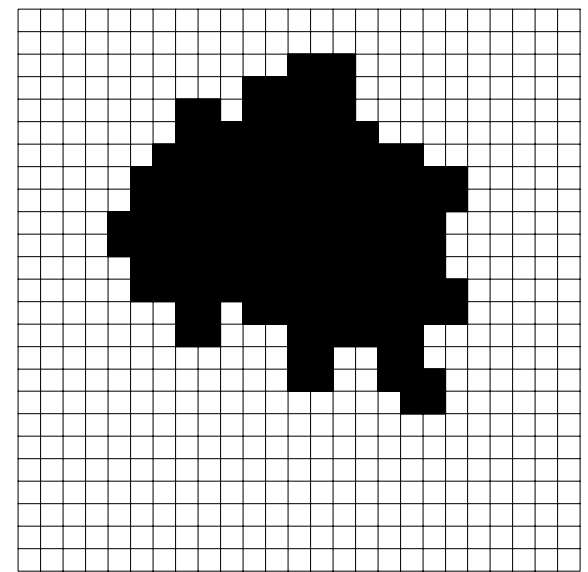

Figure 2: Schematic drawing of PEREGRINE's definition of a block. A block is defined as a mask array of square pixels which are either blocked or open. The mask array is currently defined to be a $500 \times 500$ array corresponding to physical dimensions of $25 \times 25 \mathrm{~cm}^{2}$ at axis. This $0.5 \mathrm{~mm}$ resolution is demagnified to $0.3 \mathrm{~mm}$ for typical block tray positions.

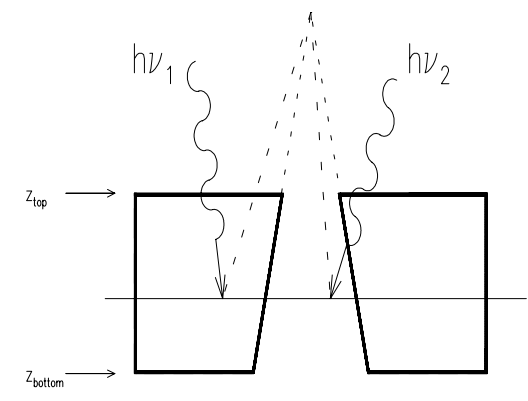

Figure 3: Schematic drawing of how PEREGRINE determines if a photon is within the block material or within the open portion. The mask array shown in Figure 2 is translated (and scaled) to be at the photon's current position. If the photon's $(\boldsymbol{x}, \boldsymbol{y})$ location corresponds to a blocked pixel, then the photon is within the block itself. If the pixel is open, the photon is in an air-filled region.

\section{Physics Methods}

Photon transport through patient-dependent beam modifiers can be done with a full-physics propagation of the photons through these beam modifiers, transporting the resulting radiation to the patient, and then proceeding with the dose calculation. The computer time required for the transport through the wedges and blocks represents a large fraction of the overall time required for the dose calculation, and thus detracts from the goal of a fast Monte Carlo calculation. We are considering methods to increase the speed of this process.

To date we have considered three choices for photon transport through patient-dependent beam modifiers: full physics (where secondary electrons and photons may be created and followed), simple attenuation (where the photons are attenuated according to the interaction cross-section), and photon-only physics (where secondary photons but not secondary electrons may be created and tracked through the material). To assess the applicability of these approximations to photon-based dose calculations, we ran a series of MCNP [4] calculations using monoenergetic photon pencil beams of $1-\mathrm{MeV}$, 2-MeV, 5-MeV, 10-MeV, 15-MeV, and 20-MeV energy on Fe slabs of $1-\mathrm{cm}$ and $5-\mathrm{cm}$ thickness, as well as on a 6-cm Cerrobend slab. The Fe slab thicknesses were chosen to be representative of wedge thicknesses for 6-MV machines. We looked at the energy that was deposited within an $11^{\circ}$ (half-angle) cone about the pencil beam axis. This cone comprises those photons that pass through the slab with no change in direction or energy, as well as those photons which are scattered within the slab, but would still fall within $10 \mathrm{~cm}$ of the unscattered photons at 50-cm distance.

\section{Simple Attenuation Physics}

Figure 4 shows the fraction of the direct radiation penetrating the slab to the total radiation (direct and scattered) for the two Fe slabs and the Cerrobend slab. The direct component represents $98 \%$ of the total transmitted energy for the $1-\mathrm{cm} \mathrm{Fe}$ slab, between $86 \%$ to $91 \%$ of the total transmitted energy for the $5-\mathrm{cm} \mathrm{Fe} \mathrm{slab,} \mathrm{and} \mathrm{between}$ $76 \%$ and $86 \%$ for the Cerrobend slab.

This suggests that the simple attenuation model (in which there is no scattered radiation) would systematically underestimate the energy fluence through beam modifiers such as wedges and blocks. This underestimation would be energy-dependent and materialthickness-dependent (a problem for wedges). We therefore reject this approximation.

\section{Photon-Only Physics}

The next approximation studied was photon-only physics. In this approximation, the photons are allowed to scatter, but we do not track any electrons that may be created. This is a more realistic approximation than simple attenuation, but it neglects the possibility that secondary electrons can produce photons, which in turn could reach the patient. Results of these calculations are shown in Figure 5. The photon-only physics approximation accurately reproduces the amount of energy that passes unscattered through each of the slabs. Comparing the middle and bottom panels of Figures 4 and 5, we find that the photon-only approximation underestimates the amount of scattered radiation that would reach the patient. We see, however, that the amount of scattered radiation is well reproduced up to $5 \mathrm{MeV}$ for the materials studied. Above this energy, the effect of neglecting processes $\gamma \Rightarrow \boldsymbol{e} \Rightarrow \gamma$ becomes noticeable for the $5-\mathrm{cm}$ Fe slab, and pronounced for the Cerrobend case.

\section{Current PEREGRINE Practice}

As shown in Figures 4 and 5, photon-only physics appears to be an adequate approximation for photon transport through materials of general interest, to at least $5 \mathrm{MeV}$ photon energy. Above this energy the amount of scattered radiation that would reach the patient is increasingly underestimated.

The errors for the monoenergetic pencil beams do not correspond to errors for a photon machine run at the equivalent electron beam energy. If we calculate the energy fluences for the polyenergetic spectra which are typical for photon machines (Figure 6) and estimate the transmission through the various slabs, we find that the total fluence error, for the Fe slabs, is less than $1 \%$ even for the 18MV distribution (Figure 7). For the Cerrobend slab the error is approximately $2 \%$ for the 18 -MV distribution. In a normal treatment this $2 \%$ error is in the shaded region of the blocked field.

Because the photon-only approximation appears to be adequate in the photon energy range of interest and adequate for the materials of interest, we have installed it into the current version of PEREGRINE. This allows us to use wedges and other patient modifiers for testing and development work. 


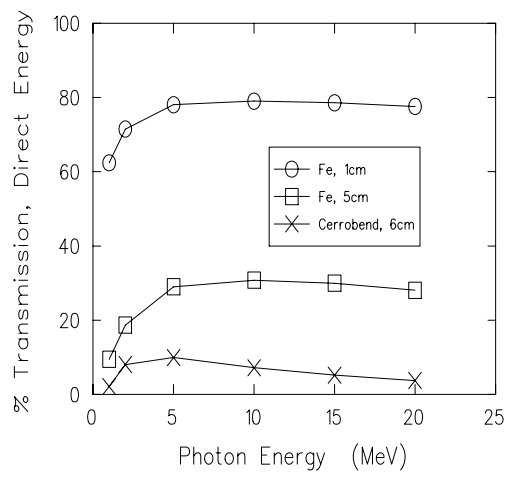

(a)

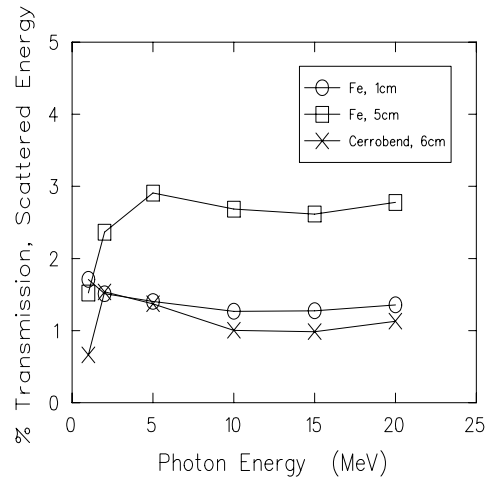

(b)

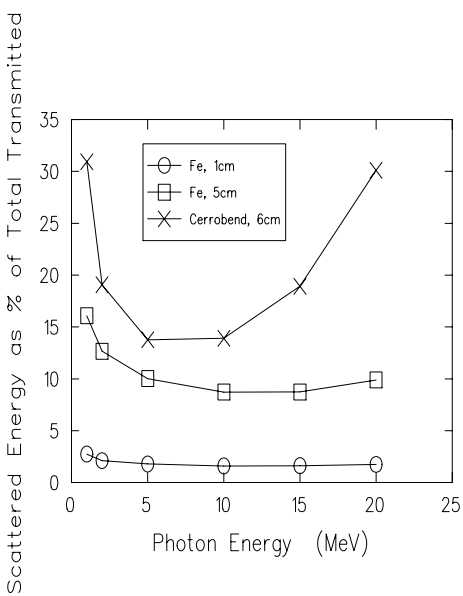

(c)

Figure 4: Full-physics calculations for monoenergetic transmission through slabs of various materials. These calculations are used to validate reduced-physics approximations. (a) Percentage of incident energy which is unscattered as it passes through the material. (b) Percentage of incident energy which is scattered by the material, but which would still land within $10 \mathrm{~cm}$ of the unscattered rays at the patient. (c) The scattered radiation as a percentage of the total radiation reaching the patient.

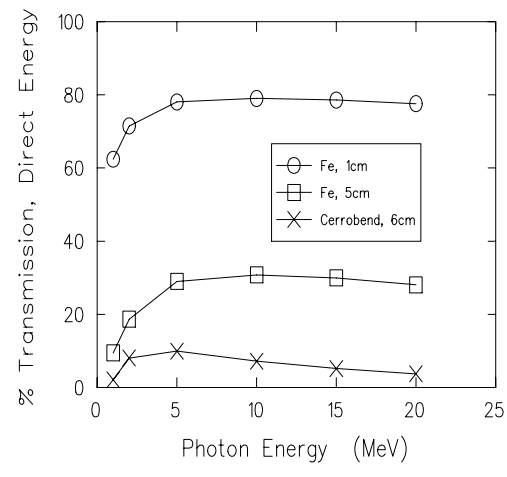

(a)

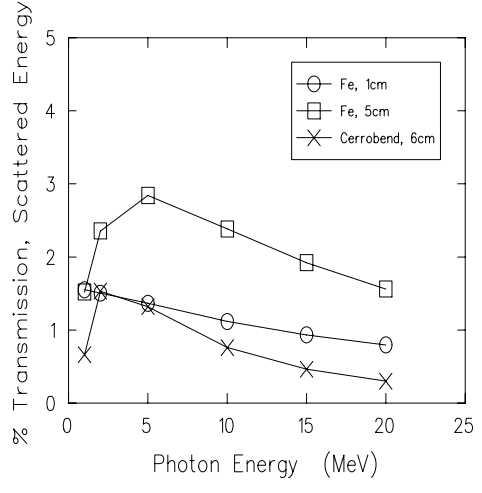

(b)

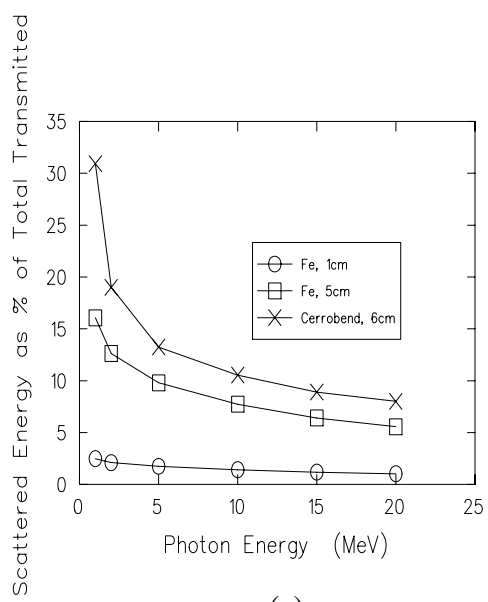

(c)

Figure 5: Photon-only physics calculation for monoenergetic transmission through slabs of various materials. (a) Percentage of incident energy which is unscattered as it passes through the material. (b) Percentage of incident energy which is scattered by the material, but which would still land within $10 \mathrm{~cm}$ of the unscattered rays at the patient. (c) The scattered radiation as a percentage of the total radiation reaching the patient. In the photon-only approximation, however, this percentage is underestimated (compare with Figure 4).

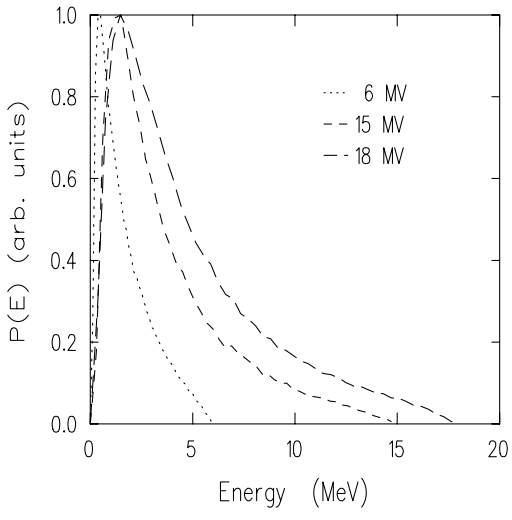

Figure 6: Energy distribution curves for $6 \mathrm{MV}, 15 \mathrm{MV}$, and $18 \mathrm{MV}$ photon machines.

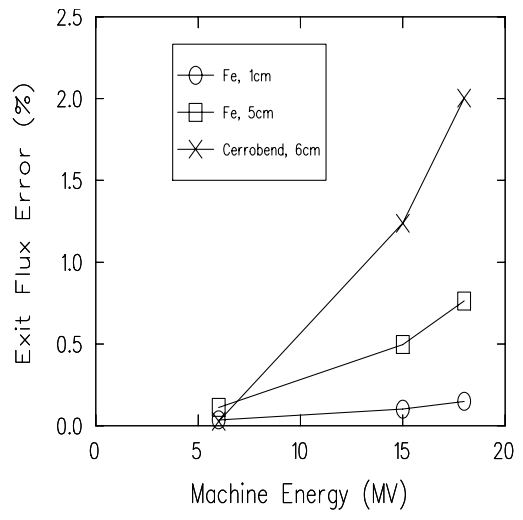

Figure 7: Fluence errors based on calculated energy distributions for 6,15 , and $18-\mathrm{MV}$ machines. 


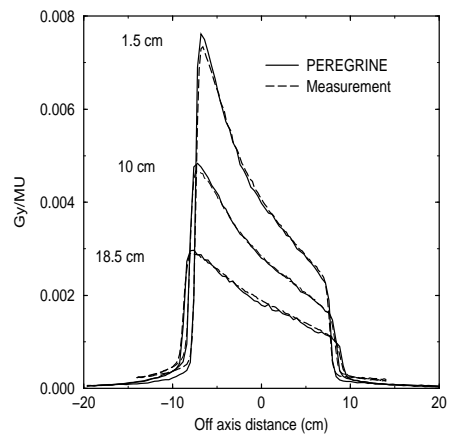

Figure 8: PEREGRINE calculation of profiles for a $60^{\circ}$ wedged $15 \times 15-\mathrm{cm}^{2}$ field (6-MV machine)

As a sample of the viability of the photon-only approximation, we show results from a PEREGRINE calculation of a $60^{\circ}$ wedged $15 \times 15 \mathrm{~cm}^{2}$ field. The plots in Figure 8 show good agreement between calculations and experimental data.

\section{Summary}

We have implemented algorithms in PEREGRINE to treat blocks and wedges in a geometrically simple fashion. We have investigated the level of sophistication required for the physics involved to get accurate results without the CPU time penalty of full-physics particle tracking. Photon-only physics gives reasonable agreement for polyenergetic clinical photon beams up to $18 \mathrm{MV}$. Studies using monoenergetic beams suggest that this approximation may be inadequate for higher energies. Work continues to find approaches that will give better agreement at these higher energies without the computational burden of full-physics tracking.

\section{References}

1. C.L. Hartmann Siantar, et al., 1997. This volume.

2. B. Libby, et al., 1997. This volume.

3. L.J. Cox, et al., 1997. This volume.

4. J. F. Briesmeister, $M C N P^{\mathrm{TM}}$ - A General Monte Carlo N-Particle Transport Code Version 4A, Report LA-12625, (Los Alamos National Laboratory, Los Alamos, NM, 1988). 


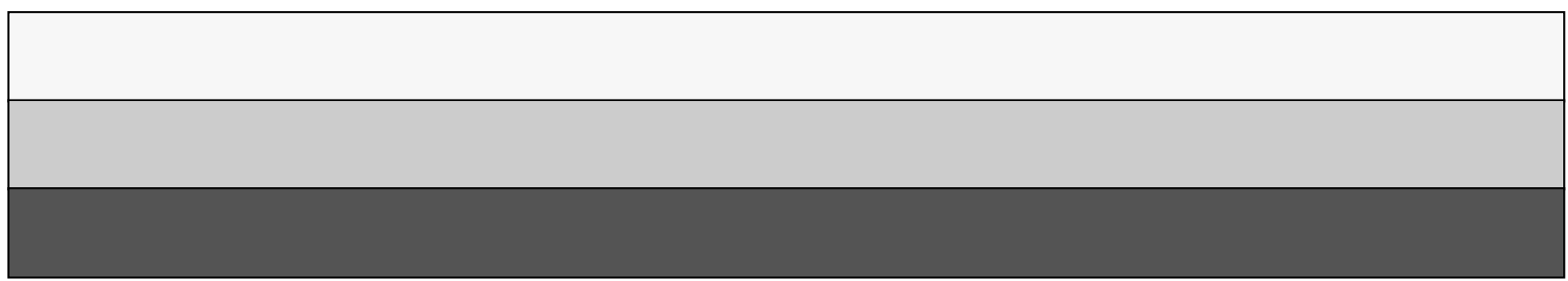

